OPEN ACCESS

Edited by:

Raul Antonio Sperotto,

Centro Universitário Univates, Brazil

Reviewed by:

Oswaldo Valdes-Lopez, National Autonomous University of Mexico, Mexico Koichi Kobayashi, University of Tokyo, Japan

${ }^{*}$ Correspondence: Fangsen Xu fangsenxu@mail.hzau.edu.cn

Specialty section: This article was submitted to Plant Nutrition, a section of the journa

Frontiers in Plant Science

Received: 01 November 2016 Accepted: 09 January 2017

Published: 25 January 2017

Citation:

Du $H$, Yang $C$, Ding G, Shi L and Xu F (2017) Genome-Wide Identification and Characterization of SPX Domain-Containing Members and Their Responses to Phosphate Deficiency in Brassica napus. Front. Plant Sci. 8:35 doi: 10.3389/fpls.2017.00035

\section{Genome-Wide Identification and Characterization of SPX Domain-Containing Members and Their Responses to Phosphate Deficiency in Brassica napus}

\author{
Hongyuan Du ${ }^{1,2}$, Chang Yang ${ }^{2}$, Guangda Ding ${ }^{2}$, Lei Shi1,2 and Fangsen $\mathrm{Xu}^{1,2 *}$ \\ ' National Key Laboratory of Crop Genetic Improvement, Huazhong Agricultural University, Wuhan, China, ${ }^{2}$ Key Laboratory \\ of Arable Land Conservation, Ministry of Agriculture, Huazhong Agricultural University, Wuhan, China
}

The importance of SPX domain-encoding proteins to phosphate (Pi) homeostasis and signaling pathways has been well-documented in rice and Arabidopsis. However, global information and responses of SPX members to P stress in allotetraploid Brassica napus, one of the world's major oil crops that is sensitive to $P$ deficiency, remain undefined. We identified a total of 69 SPX domain-containing genes in the B. napus genome. Based on the domain organizations, these genes were classified into four distinct subfamilies - SPX (11), SPX-EXS (43), SPX-MFS (8), and SPX-RING (7)-that represented clear orthologous relationships to their family members in Arabidopsis. A cis-element analysis indicated that $2 \sim 4$ P1BS elements were enriched in the promoter of SPX subfamily genes except BnaSPX4S. RNA-Seq analysis showed that BnaSPX genes were differentially expressed in response to Pi deficiency. Furthermore, quantitative real-time reverse transcription PCR revealed that nine SPX subfamily genes were significantly induced by $\mathrm{Pi}$ starvation and recovered rapidly after $\mathrm{Pi}$ refeeding. A functional analysis of two paralogous BnaSPX1 genes in transgenic Arabidopsis indicated their functional divergence during long-term evolution. This comprehensive study on the abundance, molecular characterization and responses to Pi deficiency of BnaSPX genes provides insights into the structural and functional diversities of these family members in B. napus and provides a solid foundation for future functional studies of BnaSPX genes.

Highlight: The genome-wide identification and characterization of SPX genes in $B$. napus and their responses to Pi deficiency provide comprehensive insights into the structural and functional diversities of the family members in B. napus and their potential in Pi homeostasis and signaling responsiveness to Pi stress.

\footnotetext{
Keywords: Brassica napus, genome-wide analysis, SPX domain gene, Pi stress, expression profile, cis-element,
} gene function

Abbreviations: AS, acetosyringone; BLAST, basic local alignment search tool; DAPI, 4', 6-diamino-phenylindole; FPKM, fragment per $\mathrm{Kb}$ of exon model per million reads; GRAVY, the grand average of hydropathy; MEME, multiple expectation maximization for Motif Elicitation; MES, 2-( $N$-morpholino)ethanesulfonic acid hydrate; Pi, inorganic phosphate; PSI, Pi starvation-induced genes; qRT-PCR, quantitative real time PCR 


\section{INTRODUCTION}

Phosphorus $(\mathrm{P})$, an indispensable macronutrient for plant growth and development, is absorbed by root systems in the form of Pi from soil matrices (Nussaume et al., 2011). However, Pi in soils is dilute and less diffusible because it mainly exists as an integral component in $\mathrm{Ca}, \mathrm{Fe}$ or $\mathrm{Al}$ salts, or in the form of organic molecules (Raghothama and Karthikeyan, 2005). Therefore, plants often encounter a scarcity of Pi in soils in both agricultural and natural contexts. However, plants have gained series of evolutionarily adapted strategies to actively interact with Pi stress (Vance et al., 2003; Morcuende et al., 2007; Jain et al., 2012). Unveiling the molecular mechanism underlying the Pi starvation responses of plants would be pivotal for developing crop plants with enhanced $\mathrm{Pi}$ acquisition and better $\mathrm{P}$ use efficiency under low Pi conditions. Considerable information has been gathered on the components of the Pi starvation signaling pathway that has been well reviewed in the past decade (Chiou and Lin, 2011; Wu et al., 2013; Zhang et al., 2014; Baker et al., 2015); increasing studies have established the importance of SPX domain-containing proteins in Pi homeostasis and Pi signaling. To date, the identification and characterization of SPX members has been completed in many plant species including Arabidopsis (Duan et al., 2008), soybeans (Yao et al., 2014a), common beans (Yao et al., 2014b), and rice (Wang et al., 2009, 2014; Liu et al., 2010; Shi et al., 2014).

The SPX domain (Pfam PF03105) was named after the Suppressor of Yeast gpal (Syg1), the yeast Phosphatase 81 (Pho81), and the human Xenotropic and Polytropic Retrovirus

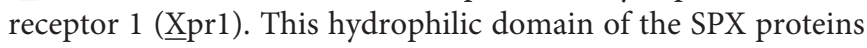
is found at the $\mathrm{N}$-termini of various proteins. In plants, proteins harboring the SPX domain are classified into four groups based on the presence of additional structural domains, namely, the SPX, SPX-EXS, SPX-MFS, and SPX-RING subfamilies (Secco et al., 2012). There are 20 and 14 SPX members in Arabidopsis and rice, respectively, which are different in structure, expression, protein subcellular location, and gene function (Duan et al., 2008; Secco et al., 2012).

Proteins exclusively harboring the SPX domain are referred to as SPX subfamily members. In Arabidopsis and rice, the SPX subfamily consists of four and six members, named AtSPX1AtSPX4 and OsSPX1-SPX6, respectively (Duan et al., 2008; Secco et al., 2012). All SPX genes, with the exception of AtSPX4 and OsSPX4, are PSI. The major roles of SPX subfamily genes are to modulate the activities of PHR1 in the regulation of PSI gene transcription, either by controlling the translocation of PHR1 from the cytoplasm to the nucleus (Lv et al., 2014) or by competing with the P1BS element for binding to PHR1 (Puga et al., 2014; Wang et al., 2014).

The second subfamily of SPX domain-containing proteins is associated with the EXS domain (PF03124), which is embedded in a hydrophobic region and named after the yeast ERD1, the human X XR1 and the yeast $\underline{S} Y G 1$ proteins (Hamburger et al., 2002). The PHO1 family members are the only proteins in eukaryotes that contain both the SPX and EXS domains (Wang et al., 2004). Transient expression analysis in Nicotiana benthamiana indicated that the EXS domain of PHO1 is essential for Pi export activity and proper localization to the Golgi and trans-Golgi network, although the EXS domain by itself cannot mediate Pi export (Wege et al., 2016). The genome of Arabidopsis contains 11 members of the PHO1 gene family (designated as PHO1 and PHO1; H1 to PHO1; H10). Diverse tissue expression patterns implied a broad role for the PHO1 gene family. However, to date, only AtPHO1 and AtPHO1;H1 have been studied and found to be involved in Pi homeostasis (Stefanovic et al., 2007, 2011). Bioinformatics and phylogenetic analysis showed that the rice genome has three $\mathrm{PHO} 1$ homologs that cluster with AtPHO1 and AtPHO1;H1. In contrast to the Arabidopsis PHO1 gene family, all three rice PHO1 genes have a cis-natural antisense transcript located at the $5^{\prime}$ end of the genes. OsPHO1;2 played a key role in the transfer of Pi from roots to shoots in rice and could be regulated by its cis-natural antisense transcripts (Secco et al., 2010).

The third subfamily of SPX domain-possessing proteins is the SPX-MFS (Major Facility Superfamily) family (PF07690). There are three SPX-MFS members in the genomes of both Arabidopsis and rice, namely SPX-MFS1 to SPX-MFS3, which are also designated the PHOSPHATE TRANSPORTER 5 (PHT5) family in Arabidopsis (Secco et al., 2012; Wang et al., 2012; Liu et al., 2016). Overexpression of PHT5 leads to Pi over accumulation and retarded growth. Based on ${ }^{31} \mathrm{P}$-magnetic resonance spectroscopy analysis, Arabidopsis pht5;1 loss-offunction mutants accumulate less Pi and exhibit a lower vacuolarto-cytoplasmic Pi ratio than controls. OsSPX-MFS1 and OsSPXMFS3 were suppressed by Pi deficiency, whereas OsSPX-MFS2 was induced (Lin et al., 2010). Heterologous complementation of a yeast mutant impaired in Pi transport indicated the capacity of OsSPX-MFS1 to transport Pi. Mutated OsSPX-MFS1 alters Pi remobilization in rice (Wang et al., 2012). OsSPX-MFS3 is a low affinity Pi transporter that mediates Pi efflux from the vacuole into the cytosol and is coupled to proton movement (Wang et al., 2015).

The fourth SPX protein subfamily contains an additional domain named the Really Interesting New Gene (RING) finger domain, a specialized type of zinc finger domain that is involved in the mediation of protein-protein interactions. The presence of a RING finger domain is a characteristic of RING-class E3 ubiquitin protein ligases, which are capable of transferring ubiquitin from an E2 enzyme to a substrate protein. Only two proteins in both rice and Arabidopsis possess the RING and SPX domains (Secco et al., 2012). To date, the only characterized member of the SPX-RING family is also called Nitrogen Limitation Adaptation (NLA), first identified by its role in nitrogen starvation resistance (Peng et al., 2007). Later, a combination of physiology and genetic studies demonstrated that NLA regulates nitrate-dependent Pi homeostasis in Arabidopsis (Kant et al., 2011). Phosphate analysis revealed that the atnla mutant showed increased Pi uptake and Pi content, especially under low-nitrate and high-phosphate availability, relative to WT plants.

Allotetraploid rapeseed (Brassica napus, $\mathrm{A}_{n} \mathrm{~A}_{n} \mathrm{C}_{\mathrm{n}} \mathrm{C}_{n}, 2 n=38$, $840 \mathrm{Mb}$ ) originated from a natural hybridization between $B$. rapa $\left(\mathrm{A}_{\mathrm{r}} \mathrm{A}_{\mathrm{r}}, 2 n=20,312 \mathrm{Mb}\right)$ and $B$. oleracea $\left(\mathrm{C}_{o} \mathrm{C}_{o}, 2 n=18\right.$, 
$540 \mathrm{Mb}$ ) approximately 7,500-12,500 years ago (Nagaharu, 1935; Chalhoub et al., 2014). Through long-term evolution and domestication, rapeseed has become second-leading crop source of vegetable oil (following soybean) for human consumption, with a total production of over 60 million tons worldwide (Bancroft et al., 2011). Although SPX domain-containing proteins have been characterized in well-studied model plants, such as rice and Arabidopsis, systematic descriptions of evolution, distribution and origin of the SPX gene family have not been conducted in $B$. napus, which is sensitive to $\mathrm{P}$ deficiency. In this study, we first identified all putative SPX genes in the $B$. napus genome, systematically characterized their structural features and conserved domains, and then provided RNA-Seqbased gene expression profiles and qRT-PCR data for BnaSPX genes both in the roots and shoots of $B$. napus in response to Pi stress. Our analyses provide the framework for future functional studies of this important gene family and the basis for exploiting candidate genes for genetic engineering of $\mathrm{P}$ efficiency in B. napus.

\section{MATERIALS AND METHODS}

\section{Identification of SPX Genes in B. napus}

SPX members were identified in B. napus based on homology with the 20 known SPX protein sequences from Arabidopsis (Secco et al., 2012) using the BLAST search program in the CNS - Genoscope database ${ }^{1}$ (Chalhoub et al., 2014). After removing redundant sequences and incomplete ORF sequences, SMART tools ${ }^{2}$ (Letunic et al., 2014), NCBI Conserved Domain Search database ${ }^{3}$ (Marchler-Bauer et al., 2015), and InterProScan ${ }^{4}$ (Mitchell et al., 2015) were used to confirm the presence of characterized domains in the candidate sequences. The putative members of the SPX family and their gene sequences were identified and defined for further analyses. The number of amino acids, CDS lengths and chromosome locations of the BnaSPX genes were obtained from the B. napus database. Physicochemical parameters, including the molecular weight $(\mathrm{kDa})$ and $\mathrm{pI}$ of each BnaSPX protein, were calculated using the compute $\mathrm{pI} / \mathrm{Mw}$ tool in $\mathrm{ExPASy}^{5}$ (Gasteiger et al., 2003). GRAVY values were calculated using the PROTPARAM tool $^{6}$ (Gasteiger et al., 2003). Subcellular location prediction was conducted using Wolf Psort ${ }^{7}$ (Hamburger et al., 2007), and the TargetP1.18 (Emanuelsson et al., 2007) server. The synteny relationships between the BnaSPXs and SPX genes in $A$. thaliana, B. rapa, and B. oleracea were evaluated using the search syntenic genes tool in BRAD $^{9}$ (Cheng et al., 2012).

\footnotetext{
${ }^{1}$ http://www.genoscope.cns.fr/brassicanapus/

${ }^{2}$ http://smart.embl-heidelberg.de/

${ }^{3}$ http://www.ncbi.nlm.nih.gov/Structure/cdd/wrpsb.cgi

${ }^{4}$ http://www.ebi.ac.uk/Tools/pfa/iprscan/

${ }^{5}$ http://www.expasy.org/tools/

${ }^{6} \mathrm{http}: / /$ web.expasy.org/protparam/

${ }^{7}$ http://www.genscript.com/wolf-psort.html

${ }^{8}$ http://www.cbs.dtu.dk/services/TargetP/

${ }^{9}$ http://brassicadb.org/brad/searchSyntenytPCK.php
}

\section{Multiple Alignment and Phylogenetic Analysis of BnaSPX Family Genes}

Multiple sequence alignment of all predicted BnaSPX protein sequences (69) and AtSPXs protein sequences (20) was performed using Clustal W software, and an un-rooted phylogenetic tree of the 89 full-length SPX protein sequences was generated with neighbor-joining (NJ) criteria in MEGA 6 (Tamura et al., 2013) with 1000 bootstrap replicates.

\section{Gene Structure and Conserved Motif Analysis of BnaSPX Members}

Multiple Expectation Maximization for Motif Elicitation ${ }^{10}$ (Bailey et al., 2009) was used to identify the conserved motif structures encoded by the BnaSPX family genes. Parameter setting included output motifs (15), minimum motif width (12), and maximum motif width (165). The exon-intron structures of the BnaSPX family genes were investigated using the online Gene Structure Display Server ${ }^{11}$ (Hu et al., 2015) based on full-length CDS alignments with corresponding genomic sequences.

\section{Plant Materials and Treatments}

The rapeseed genotype Eyou Changjia was used for gene cloning and expression analysis. The plants were grown hydroponically in an illuminated culture room with a cycle of $16 \mathrm{~h} / 24^{\circ} \mathrm{C}$ day and $8 \mathrm{~h} / 22^{\circ} \mathrm{C}$ night, and a light intensity of $300-320 \mu \mathrm{mol}$ proton $\mathrm{m}^{-2} \mathrm{~s}^{-1}$. Six-day-old seedlings germinated on gauze moistened with deionized water were transferred to nutrient solution. In addition to the $\mathrm{P}$, the complete basal nutrient solution contained $\mathrm{Ca}\left(\mathrm{NO}_{3}\right)_{2} 4.5 \mathrm{mM}, \mathrm{KNO}_{3} 4.0 \mathrm{mM}, \mathrm{MgSO}_{4}$ $2.0 \mathrm{mM}, \mathrm{H}_{3} \mathrm{BO}_{3} 46.0 \mu \mathrm{M}, \mathrm{MnCl}_{2} 9.0 \mu \mathrm{M}, \mathrm{CuCl}_{2} 0.3 \mu \mathrm{M}, \mathrm{ZnCl}_{2}$ $0.8 \mu \mathrm{M}, \mathrm{Na}_{2} \mathrm{MoO}_{4} 0.1 \mu \mathrm{M}$, and EDTA-Fe $50.0 \mu \mathrm{M}$ (Yang et al., 2011). The initial nutrient solution was $1 / 4$ full-strength for the first 5 days, then nutrient solution was replaced with halfstrength and full-strength solution at intervals of 5 days. To study gene expression patterns during Pi starvation and resupply, the seedlings were first grown under $250 \mu \mathrm{M} \mathrm{Pi}\left(\mathrm{KH}_{2} \mathrm{PO}_{4}\right)$ for 15 days after transplanting, then the seedlings were transferred to Pi-free solution, and the leaves and roots were sampled after $0,12,72$, and $240 \mathrm{~h}$. The plants grown in the Pi-free solution for 10 days $(240 \mathrm{~h})$ were retransferred to Pi-sufficient solution $(250 \mu \mathrm{M} \mathrm{Pi})$, and then the leaves and roots were harvested at 6 and $72 \mathrm{~h}$ after transferred. To analyze the expression patterns of BnaSPX members in different tissues under Pi-deficiency condition, a pot culture was performed with $10 \mathrm{mg} \mathrm{P}$ per $\mathrm{kg}$ soil. During full-bloom stage, old leaf (the lower third leaf), young leaf (the upper first leaf), bud, flowers, pods, and pod peduncle were harvested for RNA extraction. Three biological replicates were included in all experiments and all samples were frozen in liquid nitrogen and stored at $-80^{\circ} \mathrm{C}$ prior to RNA extraction.

For phenotype tests of transgenic lines, the Arabidopsis seeds were surface sterilized and cold treated at $4^{\circ} \mathrm{C}$ for 2 days. Then, the seeds were germinated on MGRL medium containing $1 \%$

\footnotetext{
${ }^{10}$ http://alternate.meme-suite.org/

${ }^{11}$ http://gsds.cbi.pku.edu.cn/
} 
(w/v) sucrose and $0.9 \%(w / v)$ agar with high $\mathrm{Pi}(1 \mathrm{mM} \mathrm{Pi}, \mathrm{HP})$ and low Pi (50 $\mu \mathrm{M} \mathrm{Pi}, \mathrm{LP})$ treatments.

\section{Expression Pattern Analysis of BnaSPXs in Response to Pi Starvation using RNA-Seq Data}

For the RNA-Seq experiment, rapeseed seedlings were grown hydroponically under $250 \mu \mathrm{M}$ Pi (high P, HP) for 15 days, then half the seedlings were moved to P-free nutrient solution $(-\mathrm{P}, 0 \mu \mathrm{M} \mathrm{P})$ and the remaining seedlings were maintained under HP conditions and used as control. The roots and leaves of every individual seedling were harvested at 10 days after the $\mathrm{P}$ was removed $\left(\mathrm{KH}_{2} \mathrm{PO}_{4}\right.$ was replaced by $\left.\mathrm{K}_{2} \mathrm{SO}_{4}\right)$. Three biological replicates were included. Total RNA was extracted from plant samples using TRIzol reagent according to the manufacturer's instructions (Invitrogen, USA). The quality and integrity of the total RNA were assessed using a NanoDrop 2000 spectrophotometer (Thermo Scientific, USA). Three microgram of RNA per sample was further processed by the purification of polyA-containing mRNA, mRNA fragmentation, doublestranded cDNA synthesis, and polymerase chain reaction (PCR) amplification using the Illumina TruSeq RNA Sample Preparation Kit (Illumina, USA) according to the manufacturer's protocol. The final cDNA libraries were sequenced on an Illumina HiSeqTM 2000 platform. For the RNA-Seq analysis based on the raw reads, clean reads were first generated by removing adapter sequences, low-quality reads and uncertain bases. A total of $643,846,484$ raw reads and $571,929,682$ clean reads were generated. The mapped reads were 496,073,698 and the unique mapped reads were $430,982,901$. So the unique mapped rate (\%) was $75.40 \%$, which were mapped to the reference genome (version $5^{12}$ ) by TopHat2 using default settings. To measure the level of gene expression, the FPKM value of each gene was calculated based on the length of the gene and the read count mapped to this gene. False discovery rate (FDR) was used in multiple hypotheses to correct the results for $P$-value. In the present study, FDR $\leq 0.05$ and the absolute value of $\log 2$ (fold-change) $\geq 1$ were used as the threshold to screen the differentially expressed genes (DEGs). A heat map of BnaSPXs gene expression was generated based on the FPKM values using the Multiexperiment View software. FPKM values based on transcriptome data are shown in Supplementary Table S3.

\section{Expression Analysis by qRT-PCR}

Total RNA was extracted from plant samples using TRIzol reagent according to the manufacturer's instructions (Invitrogen, USA). First-strand cDNA was synthesized using M-MLV reverse transcriptase and oligo (dT) according to the manufacturer's protocol (Promega, USA). Quantitative real-time RT-PCR was performed on a CFX96 Real-Time PCR Detection System (BioRad, USA) with the SYBR Green system (Toyobo, Japan). The BnaActin2 gene was used as an internal control, and the fold change was analyzed via the $2^{-\Delta \Delta C T}$ method (Livak and Schmittgen, 2001). The gene-specific primers used in the qRTPCR analysis were listed in Supplementary Table S4.

\section{Characterization of Putative Cis-elements in the Promoter Region of the SPX Genes in $B$. napus}

The 1500 bp upstream sequences relative to the translation start codon of SPX genes were downloaded from CNS - Genoscope ${ }^{12}$. The upstream 1500 bp regions of BnaSPX genes were analyzed to determine the cis-regulatory elements using the plant cis-element database PlantCARE ${ }^{13}$ Lescot et al., 2002).

\section{Vector Construction and Plant Transformation}

To construct the pBnaA2.SPX1:BnaA2.SPX1 and pBnaC3.SPX1:BnaC3.SPX1 vectors, the genomic sequences of BnaA2.SPX1 and BnaC3.SPX1, together with their upstream 1500 bp sequences, were amplified from the genomic DNA (gDNA) isolated from rapeseed genotype Eyou Changjia seedlings. The sequence-confirmed PCR fragments were inserted into the binary vector pBin35SRed using AscI and XbaI restriction enzymes. The resulting vectors carried a DsRed fluorescent protein as a selectable marker for transgenic lines (Pidkowich et al., 2007). Semi-RT-PCR was performed to validate the expression level of exogenous SPX1 in Arabidopsis wild-type (WT) Col-0 and transgenic plants by using specific primers. The primer information was present in the Supplementary Table S4.

For subcellular localization analysis, the CDSs (without the stop codon) of BnaA3.SPX1, BnaA2.SPX1, BnaC3.SPX1, BnaA3.SPX2, and BnaC3.SPX2 were PCR amplified from rapeseed cDNA and fused to the $5^{\prime}$ terminus of GFP in the vector pMDC83 via PacI and AscI restriction enzyme sites to generate 35S-BnaSPXs-GFP vectors.

The primers used for vector construction are listed in Supplementary Table S4. All constructs were mobilized into the Agrobacterium tumefaciens strain GV3101. Recombinant vectors pBnaA2.SPX1:BnaA2.SPX1 and pBnaC3.SPX1:BnaC3.SPX1 were transformed into Arabidopsis via the Agrobacterium-mediated flower dip method (Clough and Bent, 1998), and 35S-BnaSPXsGFP were used for transient expression in the leaves of tobacco (N. benthamiana).

\section{Sub-Cellular Localization Analysis}

The Agrobacterium strains harboring 35S-BnaA2.SPX1-GFP, 35S-BnaA3.SPX1-GFP 35S-BnaC3.SPX1-GFP, 35S-BnaA3.SPX2GFP, and 35S-BnaC3.SPX2-GFP, respectively, were grown to a cell density of $\mathrm{OD}_{600}=0.8$ and then harvested and resuspended in an infiltration buffer (0.5 M MES (pH5.6), $100 \mathrm{mM}$ AS and $10 \mathrm{mM} \mathrm{MgCl}_{2}$ ) to the same concentration. Then, the suspension liquid and DAPI solution $(5 \mathrm{mg} / \mathrm{mL})$ were infiltrated into the lower epidermal side of 25-day-old leaves of $N$. benthamiana plants. Two days after the injection, the GFP fluorescence and DAPI staining were observed using a confocal laser scanning microscope (TCS SP2, Leica). All fluorescence experiments were independently repeated at least three times.

\footnotetext{
${ }^{12}$ http://brassicadb.org/cgi-bin/gbrowse/B.napus_chromosome/

${ }^{13}$ http://bioinformatics.psb.ugent.be/webtools/plantcare/html/
} 


\section{Quantification of Total P}

The wild type and transgenic lines were raised in petri plates containing HP medium $(1 \mathrm{mM})$ or LP $(50 \mu \mathrm{M})$ for 11 days, then the shoots and roots were harvested for measurement of total P content as described (Ramaiah et al., 2014). Three biological replicates were included in the experiment. The values are expressed as total $\mathrm{P} \mathrm{mmol}^{-1}$ tissue dry weight.

\section{Statistical Analysis}

Data were analyzed using two-way analysis of variance (ANOVA) to detect statistically significant differences in the results of dry weight, total P concentration and qRT-PCR analysis. The data generated were subjected to Tukey's test, and different letters on the histograms indicated mean values that were statistically different at $P \leq 0.05$.

\section{RESULTS}

\section{Genome-Wide Identification of SPX Members in B. napus}

Genes encoding SPX domain-containing proteins were identified in the B. napus genome by employing homology searches and domain confirmation analysis, and a total of 69 SPX genes (BnaSPXs) were identified in the genome of B. napus. Based on the presence of additional domains in protein structure, the 69 $B n a S P X$ genes were classified into four subfamilies, including SPX, SPX-EXS, SPX-MFS, and SPX-RING. Among these four subfamilies, the SPX-EXS group is the largest with 43 members, and the SPX, SPX-MFS, and SPX-RING subfamilies include 11, 8 , and 7 members, respectively. The details of all 69 rapeseed SPX members, including the number of exons, protein properties, GRAVY values and predicted subcellular locations, are listed in Supplementary Table S1. The rapeseed SPX proteins consist of 239-825 amino acids, with corresponding molecular weights ranging from 27.61 to $95.14 \mathrm{kDa}$. BnaSPXs of the SPX-EXS and SPX-MFS subfamilies contain more amino acid residues than those of SPX and SPX-RING. EXPASY analysis revealed that the SPX protein sequences largely varied in isoelectric point (pI) values ranging from 4.91 to 9.54 , but most proteins in the same subfamily have similar parameters. Almost all SPX-EXS and SPXRING proteins have relatively high isoelectric points ( $\mathrm{pI}>7$ ), whereas the remaining proteins, particularly those in the SPXMFS family, have $\mathrm{pI}<7$. GRAVY value was defined by the sum of the hydropathy values of all amino acids divided by the protein length. Except for the proteins in the SPX-MFS subfamily, nearly all of the BnaSPXs are hydrophilic, with a GRAVY value $<0$. TargetP and Wolf Psort were used to predict the subcellular location of the 69 BnaSPX proteins, which included the nucleus, plasma membrane and cytoplasm. Most of the proteins in the SPX-MFS and SPX-EXS subfamilies are located in the plasma membrane, and nearly all the members in the SPX and SPX-RING groups are exclusively located in the nucleus. The diversity in subcellular locations implies different functions within the SPX family. The 67 SPX genes (except for BnaCn.SPXMFS3 and BnaCn.PHO1; H8) are unevenly distributed on 18 of B. napus 19 chromosomes. The majority of BnaSPX genes are located on the chromosome arms that are associated with high rates of recombination. The number of SPX genes on a single B. napus chromosome ranges from 1 (A04) to 11 (A09) (Supplementary Figure S1). To get a better understanding of the BnaSPXs gene evolution mechanism, we searched for the syntenic genes of BnaSPXs gene with other Brassicaceae species. The synteny analysis demonstrated that most SPX gene family members are located in well-conserved synteny regions, and some genes were deleted or gained. These findings indicate that some genes might have been translocated into a non-syntenic region (Supplementary Table S2).

\section{Sequence Alignment and Phylogenetic Analysis of BnaSPX Genes}

To examine the phylogenetic relationships among the SPX domain-containing proteins in B. napus, an unrooted phylogenetic tree was constructed from alignments of the full-length SPX amino acid sequences using the NJ method (Figure 1). The phylogenetic tree confirms that BnaSPXs could be classified into four groups (subfamilies), which is consistent with the classification results obtained using homology searches as described above (Supplementary Table S1). The largest group SPX-EXS was further classified into three distinct clades (Figure 1). Clade I genes are homologous to the Arabidopsis $\mathrm{PHO}$ and $\mathrm{PHO} ; \mathrm{H1}$, the only two genes known to be involved in long-distance Pi transport (Stefanovic et al., 2007). Clade II genes are homologous to $\mathrm{PHO} 1 ; \mathrm{H} 4$, $\mathrm{PHO} ; \mathrm{H}$, $\mathrm{PHO} ; \mathrm{H} 8$, PHOl; $\mathrm{H} 9$, and $\mathrm{PHO} 1 ; \mathrm{H1O}$. Clade III genes are homologous to $\mathrm{PHO} ;$; 2 , PHO1;H3, PHO1;H5, and PHO1;H6 in Arabidopsis.

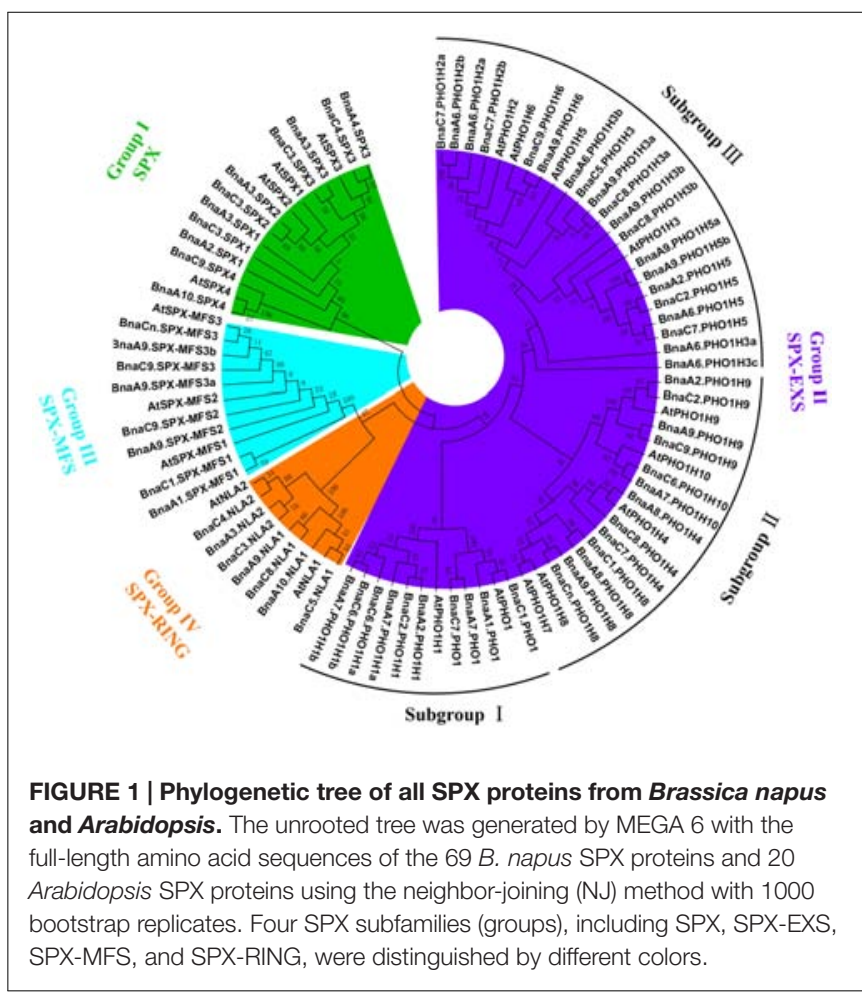




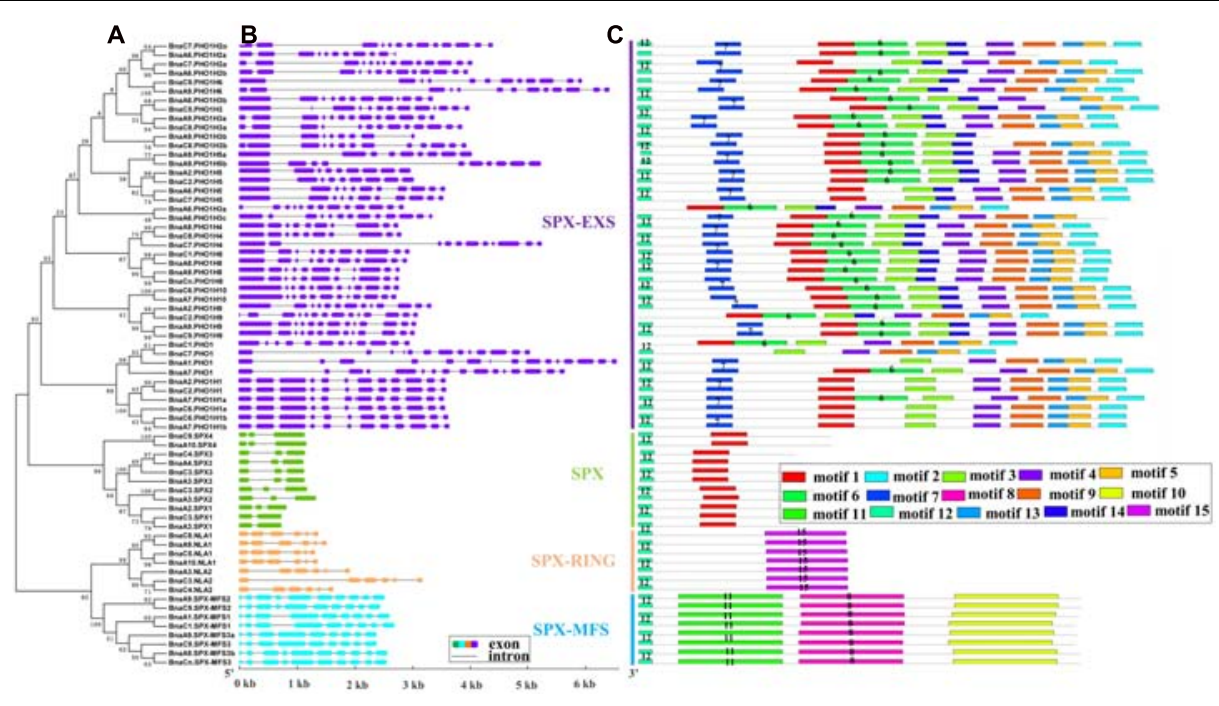

FIGURE 2 | Schematic representations of the exon-intron compositions and conserved motifs of the BnaSPX genes. (A) NJ phylogenetic tree of BnaSPXs. (B) Exon-intron organization of BnaSPX genes. Double-sided wedge boxes represent exons and gray lines represent introns. Different colors indicate different SPX gene subfamilies. The exon and intron sizes can be estimated using the scale at the bottom. (C) Distribution of conserved motifs in BnaSPX proteins. Different motifs are shown by different colors numbered 1-15. Some motifs are highlighted with different colored boxes with numbers. Lines represent protein regions without a detected motif.

\section{Structural and Conserved Motif Analysis of BnaSPX Genes}

Since the intron/exon organization and numbers are typical imprints of evolution within some gene families, we analyzed the gene structures of BnaSPXs by comparing the gDNA sequences with their corresponding coding sequences. The intron-exon structures were plotted along with the order of subfamily genes in phylogenetic tree (Figure 2A). The schematic structures revealed that each SPX gene coding sequence is disrupted by one or more introns (Figure 2B). SPX-EXS subfamily genes contain the largest number of introns, whereas SPX subfamily genes contain the fewest. The gene structures of most BnaSPXs, in terms of length and number of exons were similar to their homologous gene in Arabidopsis, especially for SPX, SPX-MFS, and SPX-RING subfamily genes. However, the genes in subfamily SPX-EXS exhibited diverse exon-intron gain/loss variations. By examining the exon-intron organization and paralogous pairs of SPXEXS genes that were clustered together at the terminal branch of the phylogenetic tree, various exon-intron changes were identified. For instance, only one out of the four BnaPHO1 genes, BnaA1.PHO1, shared the same exon-intron structure with AtPHO1, which contained 15 exons. The other three BnaPHO1 genes (BnaA7.PHO1, BnaC1.PHO1, and BnaC7.PHO1) exhibited exon-intron gain/loss variations (Figure 2B), possibly due to a single intron loss or a gain event during the long evolution process.

The conserved motifs of BnaSPX proteins were investigated further using MEME (Figure 2C), revealing a total of 15 conserved motifs (designated motifs 1-15). Motif 1, 7, and 12 specifying the conserved SPX domain indicated by the Pfam codes and WebLogo (Supplementary Figure S2) were present in almost all SPX family members. BnaA6.PHO1;H3a, BnaC1.PHO1, and BnaC2.PHO1;H9 did not show motif 12, and SPX-MFS and SPX-RING subfamily members did not contain motif 1 . The remaining motifs corresponded to the regions outside of the SPX domain region, which were distributed in specific groups in the phylogenetic tree. Proteins in the same group or subgroup contained similar motifs, whereas the motifs were divergent among different subfamilies. Conserved motifs 8, 10, and 11 specified the MFS domain and were found in all BnaSPX-MFS proteins. Motif 15 was only present in each member of the SPX-RING subfamily (Figure 2C), which indicated that this motif was specific to SPX-RING genes. Similarly, the most prominent feature of proteins in the SPXEXS group was the EXS domain, which embraced at most seven motifs, and motifs 3 and 14 were shared by all members of the SPX-EXS subfamily. These results indicated that most motifs were distributed among specific groups, which correlated with their functional divergence. Pairwise comparisons of the 69 full-length BnaSPX protein sequences revealed that amino acid sequences in the SPX-EXS group were more diverse but well conserved in the SPX-MFS subfamily (Figure 3). Otherwise, the protein sequence identity within each group was higher than that between groups.

\section{RNA-Seq-Based Expression Profiling of BnaSPX Genes during Pi Deficiency Stress}

To identify potential BnaSPX genes involved in Pi starvation signaling, we analyzed the expression profiles of all SPX genes identified in $B$. napus under Pi stress using RNA-Seq analysis. A total of 50 BnaSPX genes were detected in the shoots and/or roots at the seedling stage (Figure 4). Among these 


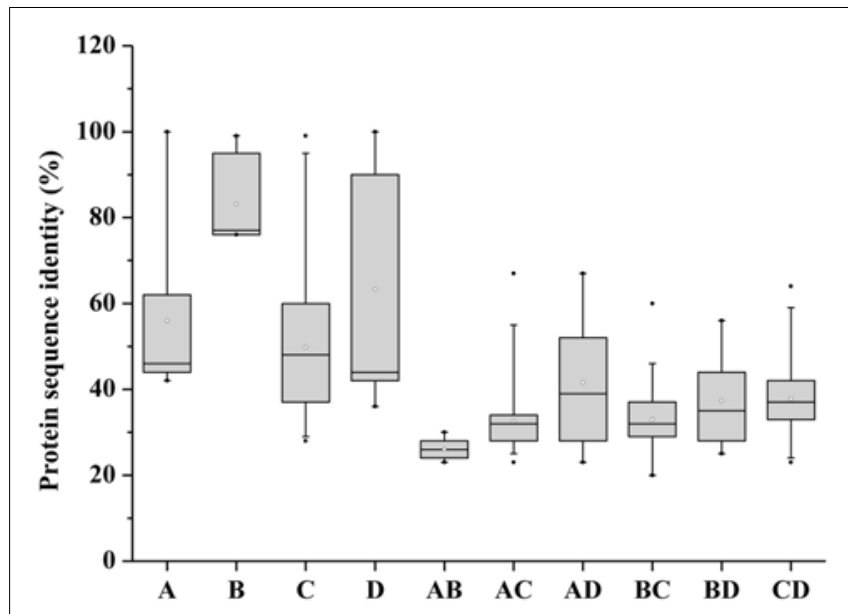

FIGURE 3 | Pairwise sequence identity of full-length BnaSPX proteins. $A, B, C$, and D represent pairwise sequence identities of the SPX, SPX-MFS, SPX-EXS, and SPX-RING groups, respectively. AB, AC, and AD represent pairwise sequence identities between SPX and SPX-MFS, SPX and SPX-EXS, and SPX and SPX-RING proteins, respectively; BC and BD represent pairwise sequence identities between SPX-MFS and SPX-EXS and SPX-MFS and SPX-RING proteins, respectively; CD represents pairwise sequence identities between SPX-EXS and SPX-RING proteins. The box plot shows the median (black line), interquartile range (box), and maximum and minimum scores (whiskers) for each data set.

BnaSPX genes, all SPX subfamily genes except BnaSPX4s were significantly up-regulated by $\mathrm{Pi}$ stress both in the shoot and root, especially for BnaA2.SPX1, BnaA3.SPX1, BnaC3.SPX2, BnaA4.SPX3, and BnaC4.SPX3, whereas SPX-MFS1s and SPX$M F S 3 s$ were moderately induced in response to Pi stress only in the shoot and not in the root. Four paralogous BnaNLA1 genes were slightly down-regulated in the root whereas BnaC4.NLA2 had no obvious change in either shoot or root when confronted with Pi stress. The expression levels of the 22 SPX-EXS subfamily genes were detected in response to $\mathrm{Pi}$ stress, among these, only $\mathrm{BnaPHO}$;H1 genes were induced with a twofold or great change in Pi stress condition (Supplementary Table S3). In contrast, two members of the BnaPHO;H3 genes, BnaA6.PHO1;H3b, and BnaC5.PHO1;H3, were mildly suppressed in one of the examined tissues (Supplementary Table S3). The gene expression profiles revealed that two BnaPHO;H5 genes, BnaA2.PHO1;H5 and BnaC2.PHO1;H5 showed diverse expression patterns in different tissues.

\section{qRT-PCR-Based Expression Profiles of BnaSPX Genes in Response to Pi Starvation}

To assess the validity and reliability of RNA-Seq data and obtain comprehensive insight into the expression profile of BnaSPXs during Pi starvation and Pi recovery, qRT-PCR was performed using gene-specific primers for 11 SPX subfamily genes. The expression profiles of all these chosen genes in 10 days were agreed with those obtained from the original RNA-Seq results $(240 \mathrm{~h})$, although the expression fold-change showed minimal differences (Figure 5). The results showed that 11 SPX subfamily

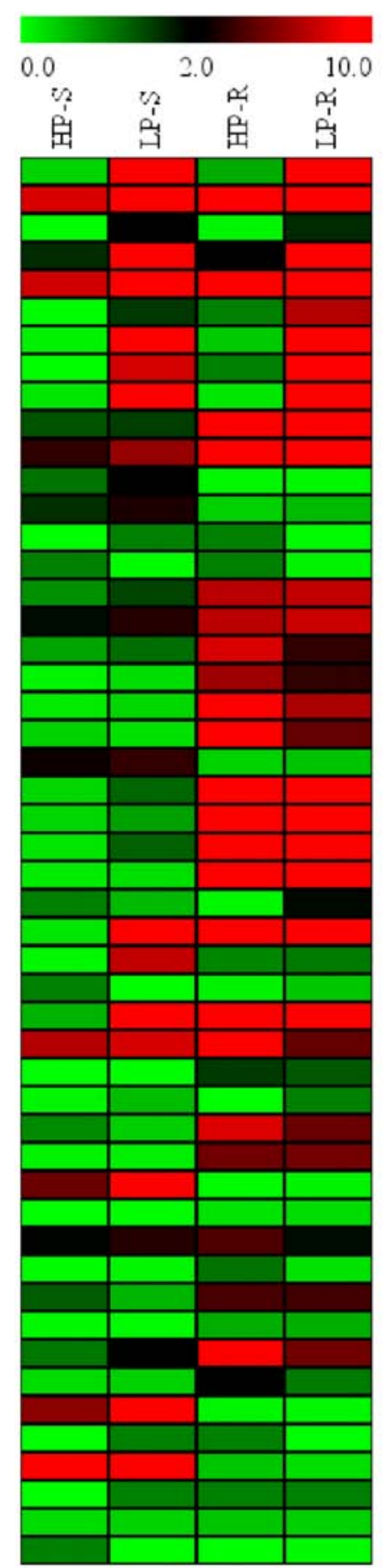

BnaA2 SPX1

BnaA3.SPX1

BnaC3.SPX1

BnaA3. SPX2

BnaC3.SPX2

BnaA3.SPX3

BnaA4 SPX3

BnaC3.SPX3

BnaC4 SPX3

BnaA10.SPX4

BnaC9 SPX4

BnaA1. SPX-MFS1

BnaC1. SPX-MFS1

BnaA9. SPX-MFS2

BnaC9. SPX-MFS2

BnaA9 SPX-MFS3a

BnaC9 SPX-MFS3

BnaA9 NLA1

BnaA10 NLA1

BnaC5.NLA1

BnaC8.NLA1

BnaC4.NLA2

BnaA1.PHO1

BnaA7.PHO1

BnaC1 PHO1

BnaC7 PHO1

BnaA7 PHO1;H1a

BnaA7. PHO1;H1b

BnaC2.PHO1; H1

BnaC6.PHO1; H1a

BnaC6.PHO1; H1b

BnaA6.PHO 1; H3b

BnaA9. PHO1;H3a

BnaA9 PHO1;H3b

BnaC5.PHO1; H3

BnaC8 PHO1;H3a

BnaC8.PHO1;H3b

BnaC7. PHO1; H4

BnaA2. PHO1;H5

BnaA6.PHO1; H5

BnaA9.PHO1;H5a

BnaA9. PHO1, H5b

BnaC2 PHO1; H5

BnaC7 PHO1;H5

BnaC1.PHO1; H8

BnaA9. PHO1; H8

BnaA8.PHO1; H8

BnaA2.PHO1;H9

BnaC6.PHO 1; H10

BnaA7. PHO 1; H10

FIGURE 4 | Expression profile of BnaSPX genes in response to $\mathrm{Pi}$ stress. Normalized gene expression values are shown in different colors that represent the relative levels of expression indicated on the scale bar. $+\mathrm{P} /-\mathrm{P}$ indicated normal $\mathrm{Pi}$ or $\mathrm{Pi}$ starvation, respectively. S/R denotes shoot or root.

genes, except for two BnaSPX4 genes, were up-regulated at most of the time points after Pi starvation treatment. Overall, the PSI expression of most BnaSPX genes was observed within $12 \mathrm{~h}$ and peaked at $240 \mathrm{~h}$. When the Pi-deprived plants were 


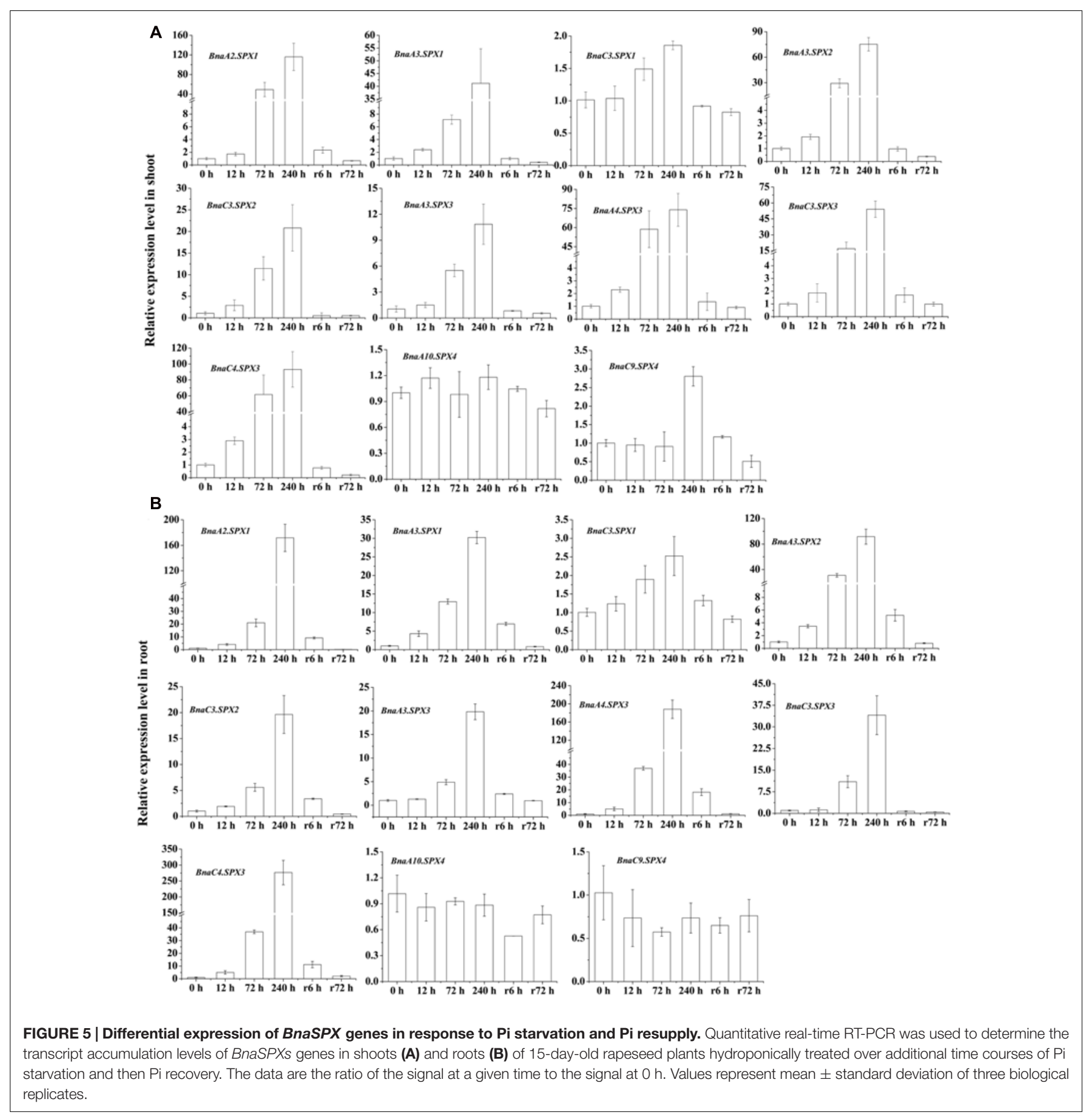

retransferred into sufficient Pi conditions, the elevated BnaSPXs transcripts were rapidly suppressed within $6 \mathrm{~h}$ and recovered to a normal level after $72 \mathrm{~h}$ of Pi-resupply. These results indicated that BnaSPXs were induced by Pi starvation continually and reversibly. We also found that some paralogs shared different induction sensitivity and intensity in response to Pi deficiency stress. Taking BnaSPX1s as an example, BnaA2.SPX1 and BnaA3.SPX1 had a high sensitivity to Pi stress by responding to Pi starvation early. Nevertheless, the Pi-starvation induction of
BnaC3.SPX1 was observed until $72 \mathrm{~h}$. In contrast, four BnaSPX3 paralogs showed a similar expression pattern to Pi deficiency; the transcript levels of all paralogous genes of BnaSPX3 exhibited a rapid and significant increase triggered by $P$ i stress.

Because most BnaSPXs in the SPX subfamily are Piinduced genes, qRT-PCR was performed to obtain an overview of the expression patterns of BnaSPX members in different tissues including old leaf (the lower third leaf), young leaf (the upper first leaf), bud, flower, pods, and pod 


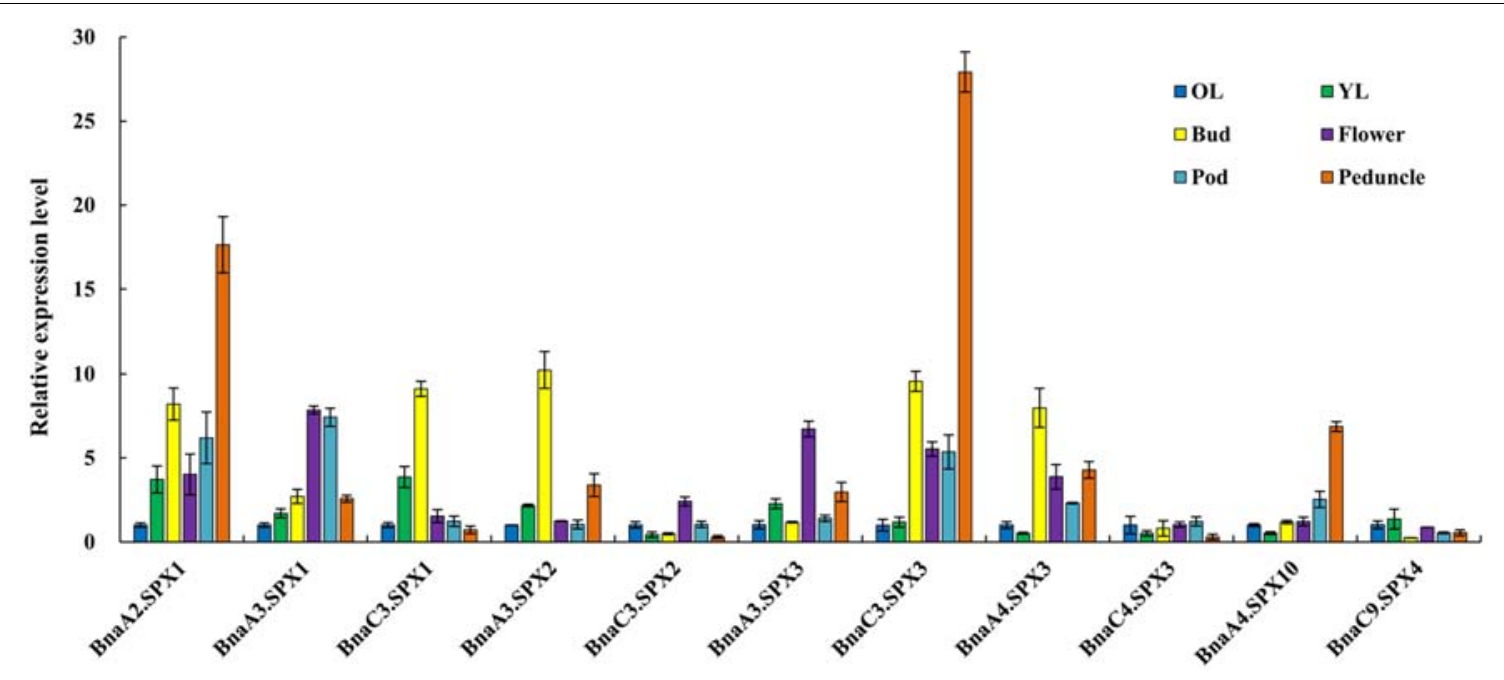

FIGURE 6 | Expression profiles of BnaSPX genes across different tissues under Pi deficiency. The relative expression of BnaSPXS was analyzed by quantitative RT-PCR in old leaves (OL), young leaves (YL), buds, flowers, pods, and pod peduncles. The expression level of BnaSPXs in old leaves was set as 1. Values represent the mean $\pm \mathrm{SD}$ of three biological replicates.

peduncle under Pi-deficiency conditions (Figure 6). Two BnaSPX1 paralogous genes (BnaA2.SPX1 and BnaA3.SPX1), in addition to two BnaSPX3 genes (BnaC3.SPX3 and BnaA4.SPX3), had predominant expression in one or more reproductive organs such as buds, flowers, pods, and pod peduncles while expressing these at low levels in vegetative tissues. BnaA10.SPX4 had preferential expression in the peduncles, whereas the BnaC3.SPX2, BnaC4.SPX3, and BnaC9.SPX4 genes were expressed at low levels in all tested tissues

\section{Cis-element Identification in the Promoter of SPX Family Genes}

Cis-regulatory elements play important roles in the global regulation of gene expression. Growing evidence indicates that genes with similar expression patterns may contain the same regulatory elements in their promoters. Many identified Pistarvation induced genes like signal molecules AtIPS1/At4, miRNAs, PTs, and purple acid phosphatases (PAPs), were found to be regulated by PHR1, the central regulator in the Pi signaling. The cis-element P1BS (PHR1 binding site), which contained an imperfect palindromic 8-bp sequence (GNATATNC) and was a conserved cis-element responding to Pi stress, showed gathered in the promoter of these PSR genes (Wu et al., 2013). The cis-elements in $66 \mathrm{BnaSPXs}$, expect for BnaA6.PHO1;H2a, BnaA9.SPX-MFS3, and BnaA9.PHO1;H3a with incomplete or low-quality promoter sequences, were analyzed using the online software PlantCARE based the $B$. napus genome data. Various types of cis-acting elements, including stress response-, hormone response-, and development-related elements, were detected in the promoter regions of BnaSPX genes (Figure 7), and most $B n a S P X$ genes contained more than one cis-element type in their promoter regions, suggesting that these BnaSPX genes may be involved in complex regulatory networks. P1BS, a conserved cis-element responding to Pi stress, was significantly enriched in the promoters of SPX subfamily genes, with the exception of Bna.SPX4s. Two or three copies of the P1BS element were present in the promoters of these genes, most of which were distributed in the proximal terminal end of the promoters. However, no or only one copy of the P1BS element was found in the promoters of the other three SPX subfamily genes, and most P1BS was distributed in the distal promoters. Interestingly, we found that the W-box element was widely distributed in the promoters of BnaPHO1;H2s, BnaPHO1;H3s, BnaPHO1;H6s, and BnaPHO1;H8s, indicating that these genes may be regulated by WRKY transcription factors.

\section{Functional Divergence of Paralogous BnaSPX Genes in Transgenic Arabidopsis}

The paralogous BnaSPX proteins were highly similar to each other, with an amino acid identity/similarity ranging from 87/89 to $99 / 99 \%$ (Supplementary Figure S3). To further investigate the putative functional divergence among the paralogous SPX genes, we first determined the subcellular localization of selected BnaSPX1s and BnaSPX2s (Figure 8). BnaSPXs- green fluorescent protein (GFP) fusion proteins driven by a $35 \mathrm{~S}$ promoter were transiently expressed in $N$. benthamiana leaves, and all the BnaSPXs-GFP fusion proteins were exclusively colocalized with the site of DAPI staining, indicating that all tested BnaSPX1s were localized in the nucleus.

Then, we examined whether the two nuclear SPX1 proteins BnaA2.SPX1 and BnaC3.SPX1, which have a relatively low protein similarity (87\%), were equally involved in Pi homeostasis. Transgenic Arabidopsis harboring the gDNA of BnaA2.SPX1 and BnaC3.SPX1 under the control of their native promoter were generated and evaluated for changes in dry weight and total $\mathrm{P}$ content under HP $(1000 \mu \mathrm{M})$ and LP (50 

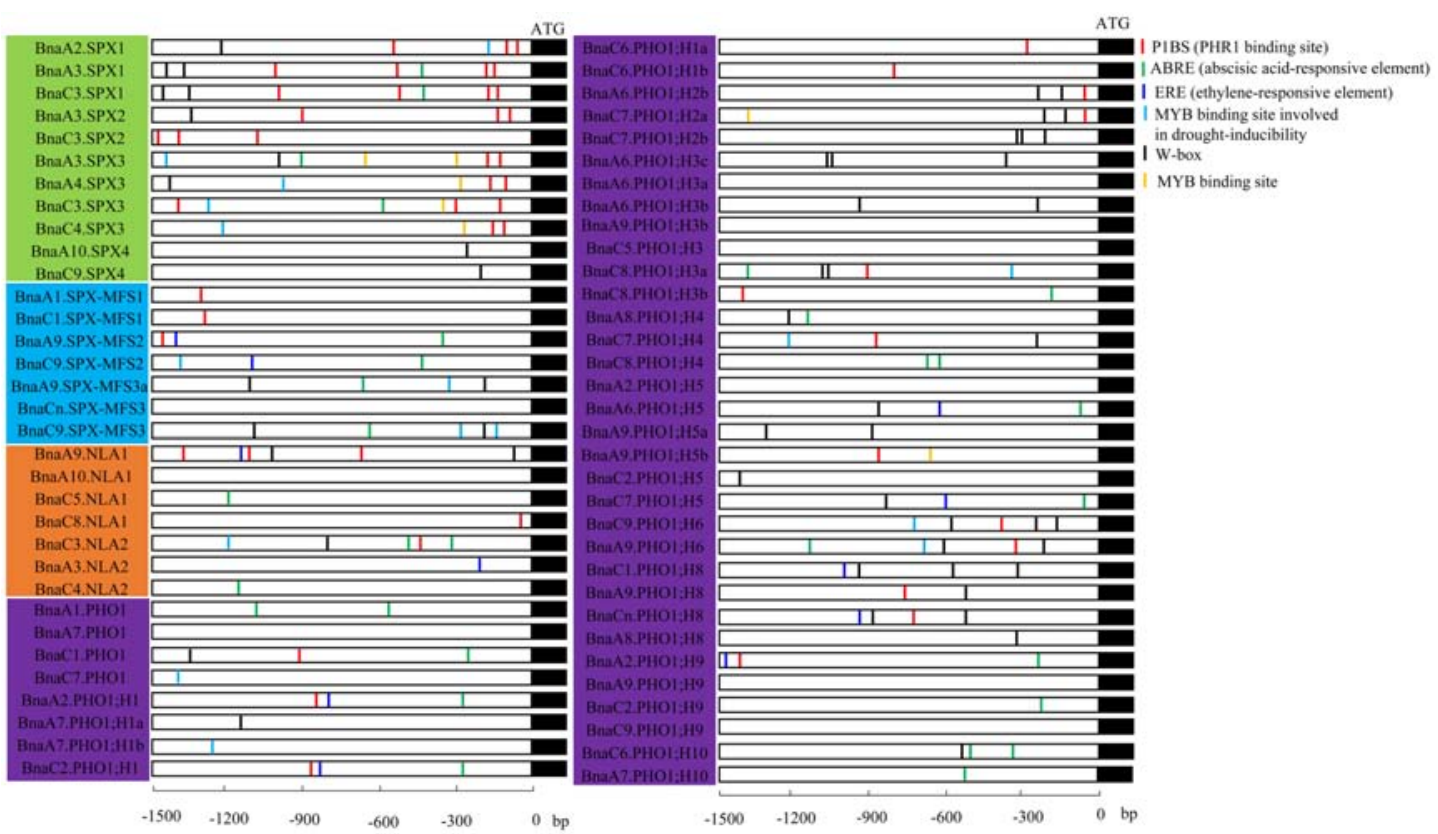

FIGURE 7 | Cis-element analysis of the promoter regions of BnaSPX genes. The micro-segments in different colors were the putative cis-element sequence. The description of the six cis-elements were in brackets. Four SPX subfamilies, including SPX, SPX-EXS, SPX-MFS, and SPX-RING, were distinguished by different background colors.

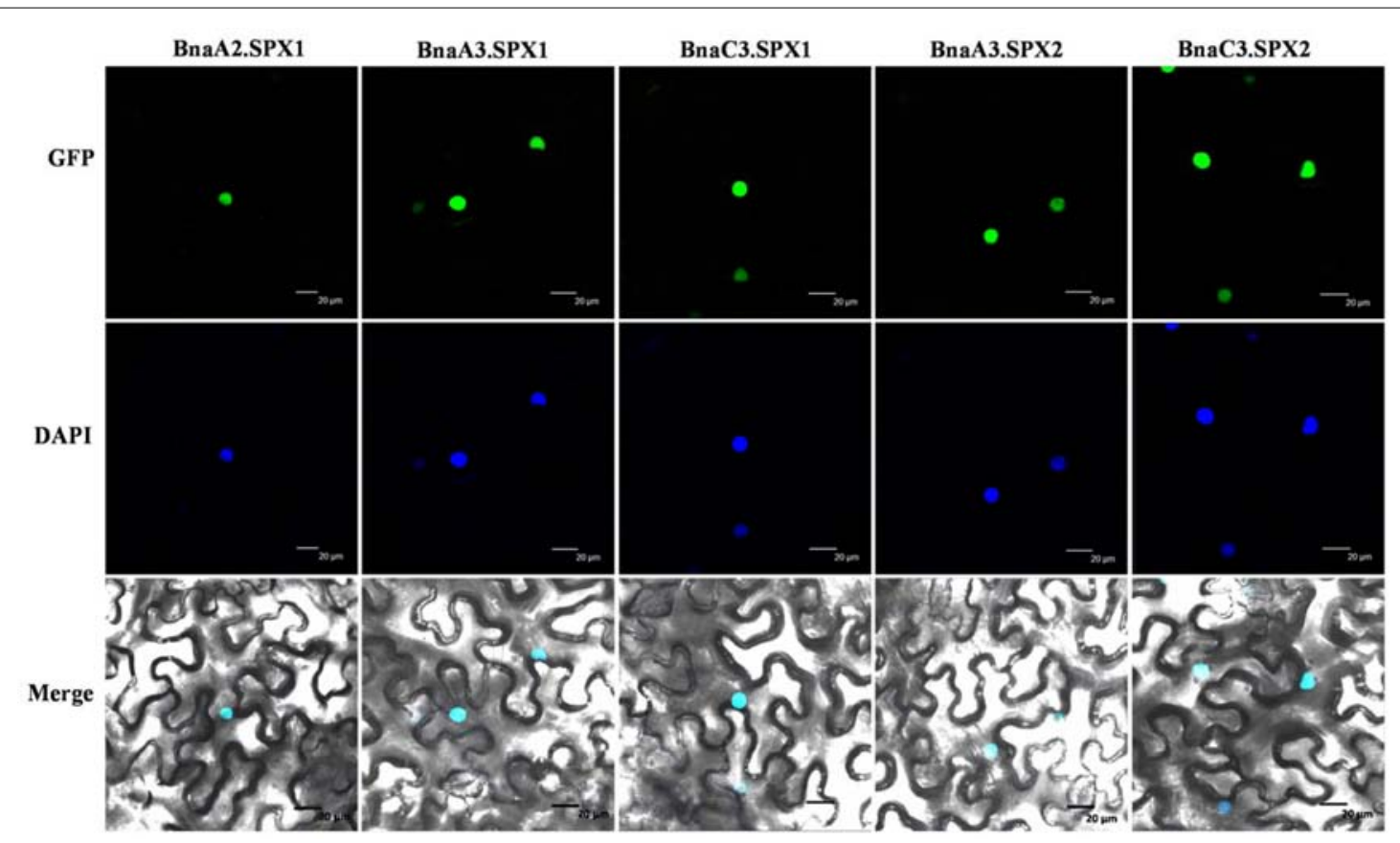

FIGURE 8 | Subcellular localization of BnaSPXs-GFP. Confocal images were taken from Nicotiana benthamiana leaf epidermal cells. The positions of the nuclei are shown by DAPI staining. Bar $=20 \mu \mathrm{m}$

$\mu \mathrm{M})$ conditions. Interestingly, transgenic lines expressing the BnaC3.SPX1 gene had no obvious phenotypic differences in comparison to the wild type despite Pi status (Supplementary Figure S4), whereas transgenic lines expressing the BnaA2.SPX1 gene displayed retarded growth and showed more sensitivity to $\mathrm{Pi}$ deficiency compared to wild-type Col-0, especially during $\mathrm{Pi}$ deficiency (Figures 9A,B), The two transgenic lines (A2.SPX1-2 and A2.SPX1-5) showed reduced plant dry 


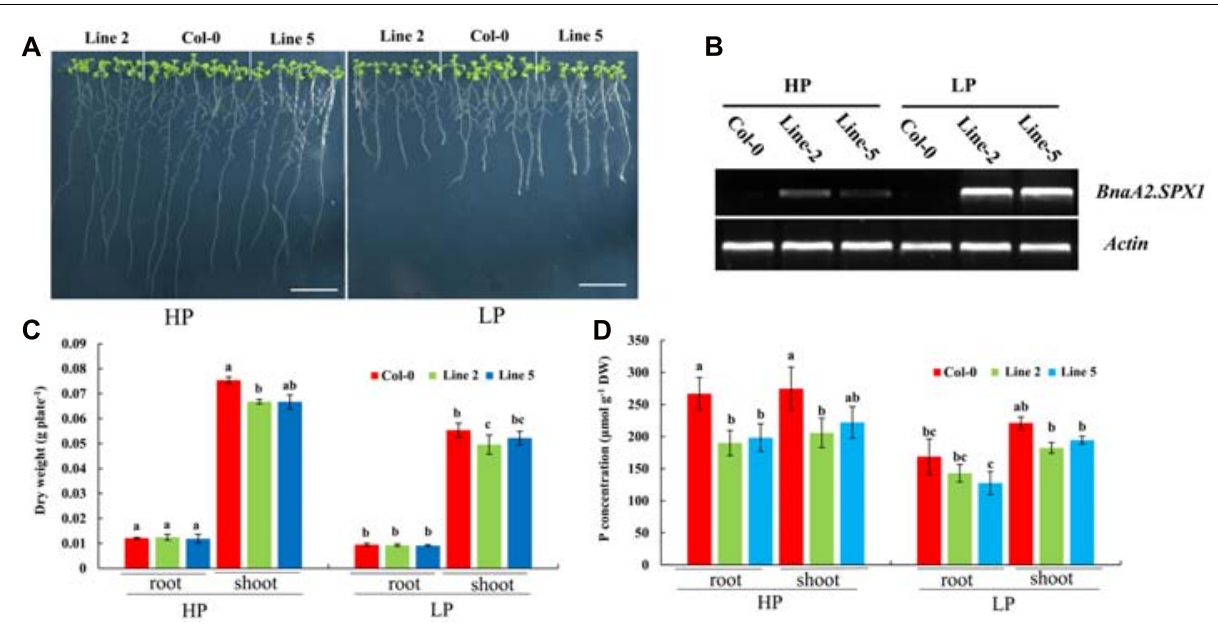

FIGURE 9 | Physiological effects and the influence on the Pi concentration of altering SPX1 activity in Arabidopsis. (A) Phenotype of wild-type (Col-0) and transgenic lines (Line-2 and Line-5) with BnaA2.SPX1 grown for 9 days under HP (1000 $\mu$ M Pi) and LP (50 $\mu$ M Pi) conditions; bar, 2 cm. (B) Semi-qRT-PCR analysis of the expression level of BnaA2.SPX1 in Col-0 and transgenic lines (Lines 2 and 5) under HP and LP conditions. Atactin7, was used as the internal control. All the experiments were repeated two times with identical results. (C,D) Dry weight (C) and total P concentration (D) in shoots and roots of WT and two transgenic lines grown under two Pi conditions. Different letters on the bar represent statistically difference at $P<0.05$.

weight and total $\mathrm{P}$ concentration relative to wild-type Col0 (Figures 9C,D). Furthermore, transgenic plant harboring pBnaA2.SPX1::BnaA2.SPX1 altered the expression of a subset of Phosphate Stress Response (PSR) genes (Figure 10B). The tested genes, including phosphate transporters (PTs), like PHT1;1, PHT1;4 and other PSR genes, SQD1, and PAP10, showed decreased expression in overexpressing lines relative to the Col-0 in Pi deficiency condition, miRNAs like miRNA399, miRNA 827 were also suppressed whereas their target gene PHO2 and NLA were slightly induced in the transgenic plants (Figure 10A).

\section{DISCUSSION}

In the present study, we performed a comprehensive search for SPX domain-containing genes throughout the B. napus genome, and a total of 69 full-length SPX genes were identified. These genes were divided into four distinct subfamilies based on the domain organization and phylogenetic analysis, which is highly consistent with the results from Arabidopsis and rice (Secco et al., 2012). We also found that different gene members from the SPX family were highly preserved after the formation of the tetraploid, showing that the genome structure has been stable, i.e., a very low rate of gene loss, which has often been proposed to be widespread during the early stage of a neopolyploid, thus contributing to rapid diversification of new plants (Jiao et al., 2011; Liu et al., 2014). In fact, for some artificial/synthetic tetraploids, chromosomal DNA and gene loss rates can be up to $15 \%$ during the first generations (Ozkan et al., 2002). Therefore, our findings show that an appreciable span of genome stability should occur for polyploids. Some polyploids may have very unstable genomes, whereas others may be stable. The $B$. napus genome seems to be on the more stable side.
Compared with the number of SPX members in A. thaliana and $O$. sativa, which contain 20 and 14 SPX genes, respectively (Secco et al., 2012), the number of SPX genes in rapeseed is remarkably high at 69 members. Copy number expansion of the SPX family in the B. napus genome has primarily occurred through genome duplication events (Chalhoub et al., 2014). Polyploidy or whole-genome duplication (WGD) has occurred multiple times throughout the evolutionary history of plants, providing raw genetic material for biological evolution and phenotypic innovation that helps organisms adapt to new and changing environments (Bowers et al., 2003). Gene structure analyses indicated that most BnaSPX genes in the same subfamily shared similar exon-intron organization, particularly within the SPX-RING group, where each member has precisely six exons. Considering the SPX-RING gene structures reported in other species, all genes in Arabidopsis and rice had six exons. The generally well-conserved exon structure in SPX-RING genes across different species highlights the possibility of conserved gene function and strict regulation of these genes. In contrast, the genes in the SPX-EXS subgroup exhibited diverse exon-intron gain/loss variations resulting from the integration or realignment of gene fragments in the SPX-EXS subgroup genes, which may play important roles for gene functional diversity (Xu et al., 2012).

\section{Exploring the Functions of BnaSPX1 Paralogs in Response to Pi Stress}

The functions of most SPX subfamily genes have been well characterized in Arabidopsis and rice ( $\mathrm{Lv}$ et al., 2014; Puga et al., 2014; Shi et al., 2014). In this study, we analyzed the gene expression patterns of BnaSPXs in responding to $\mathrm{Pi}$ stress by RNA-Seq data and qRT-PCR analysis, both results indicated that BnaSPXs in SPX subfamily were significantly involved in the Pi signaling pathway, which were in consistent with our previous study that BnSPX3;1 and BnSPX3;2 (here 

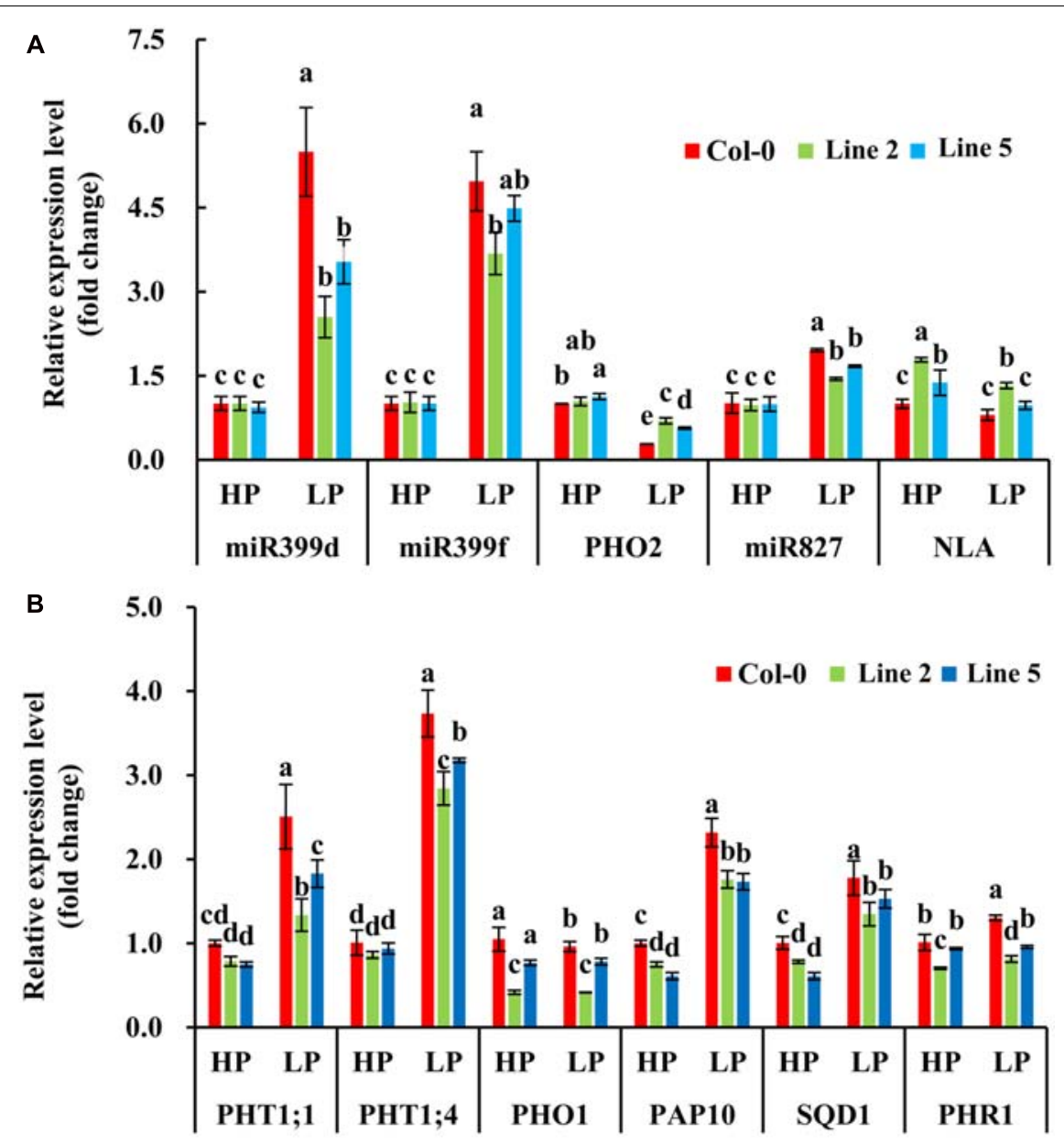

FIGURE 10 | Quantitative real time PCR analysis of the transcript accumulation levels of the components in Pi homeostasis signaling in seedlings of Col-0 and transgenic plants under two Pi conditions (A,B). Values represent mean \pm standard deviation of three biological replicates, and different letters on the bars represent statistically significant difference at $P<0.05$.

designated as BnaA3.SPX3 and BnaC3.SPX3, respectively) were $\mathrm{P}$-specific induced genes and the induction were rapid and durative during $\mathrm{Pi}$ starvation, and reversible upon resupply of Pi (Yang et al., 2011). Unlike BnaSPX3s, which were Pistarvation-specific induced genes, BnaSPX1 members expressed at a low level in normal Pi condition but induced significantly in Pi deficiency condition. We selected two BnaSPX1s as the candidates for further functional analysis in Arabidopsis by introducing BnaSPX1s into Arabidopsis under their native promoters. The expression of BnaA2.SPX1 in Arabidopsis decreased the transcript level of a subset of PSR genes (Figure 10), including PTs, SQD1, and PAP10, and miRNAs implying that BnaA2.SPX1 was a negative regulator in the Pi signaling network. Consistent results were obtained which indicated the Arabidopsis SPX1 located in nuclear and acted as a direct Pidependent inhibitor of PHR1 by inhibiting the binding to its PSI targets via P1BS motif (Puga et al., 2014). But contradictory results found that transgenic plant with 35::AtSPX1 vector in Arabidopsis enhances the transcription of several PSI (e.g., AtACP5, AtPAP2, and AtRNS1), indicating a positive regulatory role for AtSPX1 (Duan et al., 2008). Another study in rice showed that OsSPX1 is a negative regulator of OsPHR2 (the homolog of AtPHR1) and was involved in the feedback of Pisignaling network in roots that is defined by OsPHR2 and OsPHO2 (Wang et al., 2009). The molecular mechanisms of SPX regulation in Pi acquisition and translocation need to be further clarified.

We identified 3 BnaSPX1 genes (BnaA2.SPX1, BnaA3.SPX1, and BnaC3.SPX1) in B. napus by checking genes within the same taxonomic group on the phylogenetic tree. The fate of the paralogs resulted from whole genome duplication events has attracted substantial interest with respect to evolutionary novelty, species fitness and diversity. Previous studies have discussed three alternative outcomes of gene duplication: neofunctionalization, subfunctionalization, and non-functionalization (Lynch and Conery, 2000; Li et al., 2005). In the neofunctionalization model, one duplicate copy (paralog) accumulates beneficial mutations and acquires a new function, whereas the other duplicate copy retains the original gene function. In the subfunctionalization model, each paralog partitions the ancestral gene function 
(Li et al., 2005). The process of non-functionalization can occur when a redundant gene degenerates to a pseudogene or is lost from the genome due to chromosomal remodeling, locus deletion or point mutation ( $\mathrm{Gu}, 2003)$. Notably, the biochemical and functional diversity of paralogs may enable polyploids to better adapt to unfavorable environment than their diploid parents. Divergent expression patterns responding to $\mathrm{Pi}$ stress were detected among BnaSPX1 paralogs. BnaA2.SPX1 and $B n a A 3 . S P X 1$ genes were rapidly and significantly up-regulated by Pi stress, whereas the induction of BnaC3.SPX1 was delayed and slight unless long-term Pi starvation occurred (Figure 5). The expression profiles of BnaSPX1 genes across different tissues under Pi deficiency indicated that BnaA2.SPX1 and BnaA3.SPX1 had predominant expression in reproductive organs such as flowers, pod and pod peduncles, whereas BnaC3.SPX1 was expressed at low levels in the above-mentioned tissues (Figure 6). Although all the paralogous BnaSPX1 proteins are located in nuclear (Figure 8), surprisingly, only BnaA2.SPX1 negatively regulated Pi signaling; another paralog, BnaC3.SPX1, was not significantly involved in $\mathrm{Pi}$ homeostasis (Figures 9 and 10; Supplementary Figure S4). First, TF binding sites (TFBSs) or cis-elements, upstream of target genes can regulate gene expression and then alter gene function. Based on the analysis of cis-regulatory elements of the B. napus genome, there seems to be no correlation between cis-regulatory elements and expression bias among paralogous BnaSPX1s (Figure 7). In fact, all BnaSPX1 paralogs maintained the conserved P1BS elements with similar position and number in their promoters. Whether other cis-regulons exist in the promoters of these two paralogs to confer different expression bias remains to be elucidated. We also considered another possible explanation, i.e., whether epigenetic modifications such as DNA methylation and histone modification were responsible for the different $\mathrm{Pi}$ starvation responses of BnaSPX1 paralogs. It was shown that an adverse environment can induce genome-wide changes in DNA methylation status, specifically in stress-related genes in B. napus (Gao et al., 2014; Verkest et al., 2015). As such, epigenetic modification analysis between these two genes will be performed in the future.

\section{CONCLUSION}

In this study, we identified 69 SPX genes from the B. napus genome and established their classification and phylogeny using phylogenetic, gene structure, and conserved protein motif analyses. Expression profiles of BnaSPXs in response to Pi stress

\section{REFERENCES}

Bailey, T. L., Boden, M., Buske, F. A., Frith, M., Grant, C. E., Clementi, L., et al. (2009). MEME SUITE: tools for motif discovery and searching. Nucleic Acids Res. 37, W202-W208. doi: 10.1093/nar/gkp335

Baker, A., Ceasar, S. A., Palmer, A. J., Paterson, J. B., Qi, W., Muench, S. P., et al. (2015). Replace, reuse, recycle: improving the sustainable use of phosphorus by plants. J. Exp. Bot. 66, 3523-3540. doi: 10.1093/jxb/ erv210 obtained from RNA-Seq data and qRT-PCR analysis indicated that SPX subfamily genes broadly participated in the Pi signaling pathway. Characterization of Bna.SPX1 paralogs revealed their functional divergence during long-term evolution. This study provides further functional characterization of the SPX gene family in $B$. napus and provides a basis for exploiting candidate genes for genetic engineering of $\mathrm{P}$ efficiency in B. napus.

\section{AUTHOR CONTRIBUTIONS}

FX designed the study and provide guidance on the whole study. HD carried out the bioinformatic analysis, plant materials culture, qRT-PCR analysis, and drafted the manuscript. CY took parted in the plant materials culture and bioinformatic analysis. LS and GD provided the value comments and revised the grammar of the manuscript. All authors read and approved the final manuscript.

\section{ACKNOWLEDGMENT}

This study was supported by the National Natural Science Foundation of China (Grant No. 31172019).

\section{SUPPLEMENTARY MATERIAL}

The Supplementary Material for this article can be found online at: http://journal.frontiersin.org/article/10.3389/fpls.2017.00035/ full\#supplementary-material

FIGURE S1 | Distribution of BnaSPX members on B. napus chromosomes. Respective chromosome number was defined as A01 to C09 on the top of each chromosome. The size of each chromosome can be estimated from the bottom number (Mb). The 67 BnaSPXs were mapped to the 18 B. napus chromosomes, except A05. The number following each gene represents the exact chromosomal location that was available in the B. napus database.

FIGURE S2 | WebLogo plot of consensus motifs in SPX domain of BnaSPX gene family.

FIGURE S3 | Identity/similarity matrix of SPX subfamily proteins. Amino acid identity and similarity are indicated by the first and second number and the amino acid similarity and identity of paralogous SPX genes are indicated as red.

FIGURE S4 | Physiological effects of altering BnaC3.SPX1 activity in Arabidopsis. (A) The expression level of BnaC3.SPX1 in Col-0 and transgenic lines (Line 1 and Line 2) under HP (1000 $\mu \mathrm{M} \mathrm{Pi)}$ and LP (50 $\mu \mathrm{M}$ Pi) conditions by semi-qRT-PCR analysis. AtACTIN7 was used as the internal control. (B) Phenotype of wild-type (Col-0) and transgenic lines (Line-1 and Line-2) with BnaC3.SPX1 grown for 11 days under HP and LP conditions; bar, $2 \mathrm{~cm}$. 
Cheng, F., Wu, J., Fang, L., and Wang, X. (2012). Syntenic gene analysis between Brassica rapa and other Brassicaceae species. Front. Plant Sci. 3:198. doi: 10. 3389/fpls.2012.00198

Chiou, T. J., and Lin, S. I. (2011). Signaling network in sensing phosphate availability in plants. Annu. Rev. Plant Biol. 62, 185-206. doi: 10.1146/annurevarplant-042110-103849

Clough, S. J., and Bent, A. F. (1998). Floral dip: a simplified method for Agrobacterium-mediated transformation of Arabidopsis thaliana. Plant J. 16, 735-743. doi: 10.1046/j.1365-313x.1998.00343.x

Duan, K., Yi, K. K., Dang, L., Huang, H. J., Wu, W., and Wu, P. (2008). Characterization of a sub-family of Arabidopsis genes with the SPX domain reveals their diverse functions in plant tolerance to phosphorus starvation. Plant J. 54, 965-975. doi: 10.1111/j.1365-313X.2008.03460.x

Emanuelsson, O., Brunak, S., Heijne, G. V., and Nielse, H. (2007). Locating proteins in the cell using TargetP, SignalP, and related tools. Nat. Protoc. 2, 953-971. doi: 10.1038/nprot.2007.131

Gao, G., Li, J., Li, H., Li, F., Xu, K., Yan, G., et al. (2014). Comparison of the heat stress induced variations in DNA methylation between heat-tolerant and heat-sensitive rapeseed seedlings. Breed. Sci. 64, 125-133. doi: 10.1270/jsbbs. 64.125

Gasteiger, E., Gattiker, A., Hoogland, C., Ivanyi, I., Appel, R. D., and Bairoch, A. (2003). ExPASy: the proteomics server for in-depth protein knowledge and analysis. Nucleic Acids Res. 31, 3784-3788. doi: 10.1093/nar/gkg563

$\mathrm{Gu}, \mathrm{X}$. (2003). Functional divergence in protein (family) sequence evolution. Genetica 118, 133-141. doi: 10.1023/A:1024197424306

Hamburger, D., Horton, P., Park, K. J., Obayashi, T., Fujita, N., Harada, H., AdamsCollier, C. J., et al. (2007). WoLF PSORT: protein localization predictor. Nucleic Acids Res. 35, W585-W587. doi: 10.1093/nar/gkm259

Hamburger, D., Rezzonico, E., MacDonald-Comber Petétot, J., Somerville, C., and Poirier, Y. (2002). Identification and characterization of the Arabidopsis PHO1 gene involved in phosphate loading to the xylem. Plant Cell 14, 889-902. doi: $10.1105 /$ tpc.000745

Hu, B., Jin, J., Guo, A. Y., Zhang, H., Luo, J., and Gao, G. (2015). GSDS 2.0: an upgraded gene feature visualization server. Bioinformatics 31, 1296-1297. doi: 10.1093/bioinformatics/btu817

Jain, A., Nagarajan, V. K., and Raghothama, K. G. (2012). Transcriptional regulation of phosphate acquisition by higher plants. Cell. Mol. Life Sci. 69, 3207-3224. doi: 10.1007/s00018-012-1090-6

Jiao, Y., Wickett, N. J., Ayyampalayam, S., Chanderbali, A. S., Landherr, L., Ralph, P. E., et al. (2011). Ancestral polyploidy in seed plants and angiosperms. Nature 473, 97-100. doi: 10.1038/nature09916

Kant, S., Peng, M., and Rothstein, S. J. (2011). Genetic regulation by NLA and microRNA827 for maintaining nitrate-dependent phosphate homeostasis in arabidopsis. PLoS Genet. 7:e1002021. doi: 10.1371/journal.pgen. 1002021

Lescot, M., Déhais, P., Thijs, G., Marchal, K., Moreau, Y., Van de Peer, Y., et al. (2002). PlantCARE, a database of plant cis-acting regulatory elements and a portal to tools for in silico analysis of promoter sequences. Nucleic Acids Res. 30, 325-327. doi: 10.1093/nar/30.1.325

Letunic, I., Doerks, T., and Bork, P. (2014). SMART: recent updates, new developments and status in 2015. Nucleic Acids Res. 43, D257-D260. doi: 10. 1093/nar/gku949

Li, W. H., Yang, J., and Gu, X. (2005). Expression divergence between duplicate genes. Trends Genet. 21, 602-607. doi: 10.1016/j.tig.2005.08.006

Lin, S. I., Santi, C., Jobet, E., Lacut, E., Kholti, N. E., Karlowski, W. M., et al. (2010). Complex regulation of two target genes encoding SPX-MFS proteins by rice miR827 in response to phosphate starvation. Plant Cell Physiol. 51, 2119-2131. doi: $10.1093 / \mathrm{pcp} / \mathrm{pcq} 170$

Liu, F., Wang, Z. Y., Ren, H. Y., Shen, C. J., Li, Y., Ling, H. Y., et al. (2010). OsSPX1 suppresses the function of OsPHR2 in the regulation of expression of OsPT2 and phosphate homeostasis in shoots of rice. Plant J. 62, 508-517. doi: 10.1111/j.1365-313X.2010.04170.x

Liu, S., Liu, Y., Yang, X., Tong, C., Edwards, D., Parkin, I. A., et al. (2014). The Brassica oleracea genome reveals the asymmetrical evolution of polyploid genomes. Nat. Commun. 5:3930. doi: 10.1038/ncomms4930

Liu, T. Y., Huang, T. K., Yang, S. Y., Hong, Y. T., Huang, S. M., Wang, F. N., et al. (2016). Identification of plant vacuolar transporters mediating phosphate storage. Nat. Cummun. 7:11095. doi: 10.1038/ncomms11095
Livak, K. J., and Schmittgen, T. D. (2001). Analysis of relative gene expression data using real-time quantitative PCR and the 2- $\Delta \Delta$ CT method. Methods 25, 402-408. doi: 10.1006/meth.2001.1262

Lv, Q. D., Zhong, Y. J., Wang, Y. G., Wang, Z. Y., Zhang, L., Shi, J., et al. (2014). SPX4 negatively regulates phosphate signaling and homeostasis through its interaction with PHR2 in rice. Plant Cell 26, 1586-1597. doi: 10.1105/tpc.114. 123208

Lynch, M., and Conery, J. S. (2000). The evolutionary fate and consequences of duplicate genes. Science 290, 1151-1155. doi: 10.1126/science.290.5494.1151

Marchler-Bauer, A., Derbyshire, M. K., Gonzales, N. R., Lu, S. N., Chitsaz, F., Geer, L. Y., et al. (2015). CDD: NCBI's conserved domain database. Nucleic Acids Res. 43, D222-D226. doi: 10.1093/nar/gku1221

Mitchell, A., Chang, H. Y., Daugherty, L., Fraser, M., Hunter, S., Lopez, R., et al. (2015). The InterPro protein families database: the classification resource after 15 years. Nucleic Acids Res. 43, D213-D221. doi: 10.1093/nar/gku1243

Morcuende, R., Bari, R., Gibon, Y., Zheng, W., Pant, B. D., Blasing, O., et al. (2007). Genome-wide reprogramming of metabolism and regulatory networks of Arabidopsis in response to phosphorus. Plant Cell Environ. 30, 85-112. doi: 10.1111/j.1365-3040.2006.01608.x

Nagaharu, U. (1935). Genome analysis in Brassica with special reference to the experimental formation of B. napus and peculiar mode of fertilization. J. Exp. Bot. 7, 389-452.

Nussaume, L., Kanno, S., Javot, H., Marin, E., Pochon, N., Ayadi, A., et al. (2011). Phosphate import in plants: focus on the PHT1 transporters. Front. Plant Sci. 2:83. doi: 10.3389/fpls.2011.00083

Ozkan, H., Levy, A. A., and Feldman, M. (2002). Rapid differentiation of homeologous chromosomes in newly-formed allopolyploid wheat. Isr. J. Plant Sci. 50, S65-S76. doi: 10.1560/E282-PV55-G4XT-DRWJ

Peng, M., Hannam, C., Gu, H., Bi, Y. M., and Rothstein, S. J. (2007). A mutation in NLA, which encodes a RING-type ubiquitin ligase, disrupts the adaptability of Arabidopsis to nitrogen limitation. Plant J. 50, 320-337. doi: 10.1111/j.1365313X.2007.03050.x

Pidkowich, M. S., Nguyen, H. T., Heilmann, I., Ischebeck, T., and Shanklin, J. (2007). Modulating seed $\beta$-ketoacyl-acyl carrier protein synthase II level converts the composition of a temperate seed oil to that of a palm-like tropical oil. Proc. Natl. Acad. Sci. U.S.A. 104, 4742-4747. doi: 10.1073/pnas.06111 41104

Puga, M. I., Mateos, I., Charukesi, R., Wang, Z. Y., Franco-Zorrilla, J. M., Lorenzo, L. D., et al. (2014). SPX1 is a phosphate-dependent inhibitor of phosphate starvation response 1 in Arabidopsis. Proc. Natl. Acad. Sci. U.S.A. 111, 1494714952. doi: 10.1073/pnas.1404654111

Raghothama, K. G., and Karthikeyan, A. S. (2005). Phosphate acquisition. Plant Soil 274, 37-49. doi: 10.1007/s11104-004-2005-6

Ramaiah, M., Jain, A., and Raghothama, K. G. (2014). ETHYLENE RESPONSE FACTOR070 regulates root development and phosphate starvation-mediated responses. Plant Physiol. 164, 1484-1498. doi: 10.1104/pp.113.231183

Secco, D., Baumann, A., and Poirier, Y. (2010). Characterization of the rice PHO1 gene family reveals a key role for OsPHO1;2 in phosphate homeostasis and the evolution of a distinct clade in dicotyledons. Plant Physiol. 152, 1693-1704. doi: 10.1104/pp.109.149872

Secco, D., Wang, C., Arpat, B. A., Wang, Z., Poirier, Y., Tyerman, S. D., et al. (2012). The emerging importance of the SPX domain-containing proteins in phosphate homeostasis. New Phytol. 193, 842-851. doi: 10.1111/j.1469-8137.2011.04002.x

Shi, J., Hu, H., Zhang, K., Zhang, W., Yu, Y., Wu, Z., et al. (2014). The paralogous SPX3 and SPX5 genes redundantly modulate Pi homeostasis in rice. J. Exp. Bot. 65, 859-870. doi: 10.1093/jxb/ert424

Stefanovic, A., Arpat, A. B., Bligny, R., Gout, E., Vidoudez, C., Bensimon, M., et al. (2011). Over-expression of PHO1 in Arabidopsis leaves reveals its role in mediating phosphate efflux. Plant J. 66, 689-699. doi: 10.1111/j.1365-313X. 2011.04532.x

Stefanovic, A., Ribot, C., Rouached, H., Wang, Y., Chong, J. L., Belbahri, L., et al. (2007). Members of the PHO1 gene family show limited functional redundancy in phosphate transfer to the shoot, and are regulated by phosphate deficiency via distinct pathways. Plant J. 50, 982-994. doi: 10.1111/j.1365-313X.2007. 03108.x

Tamura, K., Stecher, G., Peterson, D., Filipski, A., and Kumar, S. (2013). MEGA6: molecular evolutionary genetics analysis version 6.0. Mol. Biol. Evol. 30, 27252729. doi: $10.1093 / \mathrm{molbev} / \mathrm{mst} 197$ 
Vance, C. P., Uhde-Stone, C., and Allan, D. L. (2003). Phosphorus acquisition and use: critical adaptations by plants for securing a nonrenewable resource. New Phytol. 157, 423-447. doi: 10.1046/j.1469-8137.2003. 00695.x

Verkest, A., Byzova, M., Martens, C., Willems, P., Verwulgen, T., Slabbinck, B., et al. (2015). Selection for improved energy use efficiency and drought tolerance in canola results in distinct transcriptome and epigenome changes. Plant Physiol. 168, 1338-1350. doi: 10.1104/pp.15. 00155

Wang, C., Huang, W., Ying, Y. H., Li, S., Secco, D., Tyerman, S., et al. (2012). Functional characterization of the rice SPX-MFS family reveals a key role of OsSPX-MFS1 in controlling phosphate homeostasis in leaves. New Phytol. 196, 139-148. doi: 10.1111/j.1469-8137.2012.04227.x

Wang, C., Ying, S., Huang, H. J., Li, K., Wu, P., and Shou, H. X. (2009). Involvement of OsSPX1 in phosphate homeostasis in rice. Plant J. 57, 895-904. doi: 10.1111/ j.1365-313X.2008.03734.X

Wang, C., Yue, W. H., Ying, Y. H., Wang, S. D., Secco, D., Liu, Y., et al. (2015). Rice SPX-Major Facility Superfamily 3, a vacuolar phosphate efflux transporter is involved in maintaining phosphate homeostasis in rice. Plant Physiol. 169, 2822-2831. doi: 10.1104/pp.15.01005

Wang, Y., Ribot, C., Rezzonico, E., and Poirier, Y. (2004). Structure and expression profile of the Arabidopsis $\mathrm{PHO} 1$ gene family indicates a broad role in inorganic phosphate homeostasis. Plant Physiol. 135, 400-411. doi: 10.1104/pp.103. 037945

Wang, Z. Y., Ruan, W. Y., Shi, J., Zhang, L., Xiang, D., Yang, C., et al. (2014). Rice SPX1 and SPX2 inhibit phosphate starvation responses through interacting with PHR2 in a phosphate-dependent manner. Proc. Natl. Acad. Sci. U.S.A. 111, 14953-14958. doi: 10.1073/pnas.1404680111

Wege, S., Khan, G. A., Jung, J. Y., Vogiatzaki, E., Pradervand, S., Aller, I., et al. (2016). The EXS domain of PHO1 participates in the response of shoots to phosphate deficiency via a root-to-shoot signal. Plant Physiol. 170, 385-400. doi: 10.1104/pp.15.00975
Wu, P., Shou, H. X., Xu, G. H., and Lian, X. M. (2013). Improvement of phosphorus efficiency in rice on the basis of understanding phosphate signaling and homeostasis. Curr. Opin. Plant Biol. 16, 205-212. doi: 10.1016/j.pbi.2013. 03.002

Xu, G. X., Guo, C. C., Shan, H. Y., and Kong, H. Z. (2012). Divergence of duplicate genes in exon-intron structure. Proc. Natl. Acad. Sci. U.S.A. 109, 1187-1192. doi: 10.1073/pnas.1109047109

Yang, G. Z., Ding, G. D., Shi, L., Cai, H. M., and Xu, F. S. (2011). Characterization of phosphorus starvation-induced gene BnSPX3 in Brassica napus. Plant Soil 350, 339-351. doi: 10.1007/s11104-011-0913-9

Yao, Z. F., Liang, C. Y., Zhang, Q., Chen, Z. J., Xiao, B. X., Tian, J., et al. (2014a). SPX1 is an important component in the phosphorus signalling network of common bean regulating root growth and phosphorus homeostasis. J. Exp. Bot. 65, 3299-3310. doi: 10.1093/jxb/eru183

Yao, Z. F., Tian, J., and Liao, H. (2014b). Comparative characterization of GmSPX members reveals that GmSPX3 is involved in phosphate homeostasis in soybean. Ann. Bot. 114, 477-488. doi: 10.1093/aob/ mcul47

Zhang, Z. L., Liao, H., and Lucas, W. J. (2014). Molecular mechanisms underlying phosphate sensing, signaling, and adaptation in plants. J. Integr. Plant Biol. 56, 192-220. doi: $10.1111 /$ jipb. 12163

Conflict of Interest Statement: The authors declare that the research was conducted in the absence of any commercial or financial relationships that could be construed as a potential conflict of interest.

Copyright (c) 2017 Du, Yang, Ding, Shi and Xu. This is an open-access article distributed under the terms of the Creative Commons Attribution License (CC BY). The use, distribution or reproduction in other forums is permitted, provided the original author(s) or licensor are credited and that the original publication in this journal is cited, in accordance with accepted academic practice. No use, distribution or reproduction is permitted which does not comply with these terms. 